\title{
Providing Delay Guarantees and Power Saving in IEEE 802.11e Network
}

\author{
G. Boggia, P. Camarda, F. A. Favia, L. A. Grieco, and S. Mascolo \\ DEE - Politecnico di Bari, Via E. Orabona, 4 - 70125 Bari Italy \\ \{g.boggia, camarda, f.favia, a.grieco, mascolo\}@poliba.it
}

\begin{abstract}
Recently, the 802.11e Working Group (WG) has proposed the Hybrid Coordination Function (HCF), which has a HCF Controlled Channel Access (HCCA) and an Enhanced Distributed Coordination Access (EDCA), in order to provide QoS in WLANs.

In this paper an innovative HCCA-based algorithm, which will be referred to as Power Save Feedback Based Dynamic Scheduler (PS FBDS) providing bounded delays while ensuring energy saving, has been developed. The performance of PS FBDS has been extensively investigated using ns-2 simulations; results show that the proposed algorithm is able to provide a good trade-off between QoS and power saving at both low and high network loads.
\end{abstract}

\section{Introduction}

Infrastructure 802.11 WLANs are a well assessed solution for providing ubiquitous wireless networking [1]. The building block in the architecture of such networks is the Basic Service Set (BSS), which consists of an Access Point (AP) and a set of Wireless Stations (WSTAs). The traffic from/to the WSTAs is channeled through the AP. WSTAs are usually batteries supplied devices, such as laptops or PDAs (Personal Digital Assistants), with limited lifetime [2,3]. As a consequence, the power-saving issue becomes critical and limiting for a broader diffusion of WLAN equipped hot-spots [4]. To this aim, the 802.11 standard proposes a power saving (PS) mechanism in the Distributed Coordination Function (DCF), which is based on turning off the WNIC whenever a wireless station has not any frames to transmit/receive [1]. However, several works [5-7] have highlighted that 802.11 PS presents several inefficiencies and can severely affect the frame delivering delay, thus, making the 802.11 WLANs useless for real-time applications which have specific Quality of Service (QoS) requirements. For this reason, the works [8-11] propose some optimizations of the 802.11 MAC when the PS is not used.

Moreover, the 802.11e Working Group (WG) has recently proposed a set of innovative functionalities in order to provide QoS in WLANs [12]. In particular, the core of the 802.11e proposal is the Hybrid Coordination Function (HCF), which has a HCF Controlled Channel Access (HCCA) and an Enhanced Distributed Coordination Access (EDCA). Previous works have shown that HCCA 
can be fruitfully exploited in conjunction with efficient scheduling algorithms in order to provide a bounded-delay service to real-time applications [13-15], but not considering any requirements on energy consumption. In order to bridge this gap, an innovative HCCA-based algorithm, which will be referred to as Power Save Feedback Based Dynamic Scheduler (PS FBDS), that provides bounded delays while ensuring energy saving, will be proposed in this paper. The performance of PS FBDS has been investigated using $n s-2$ simulations [16], which have shown that it is able to provide a good trade-off between QoS and power saving at both low and high network loads.

The rest of the paper is organized as follows: Section 2 gives an overview of the 802.11 MAC protocol and of QoS enhancements; Section 3 describes the theoretical background PS FBDS; Section 4 reports $n s-2$ simulation results. Finally, the last section draws the conclusions.

\section{The IEEE 802.11 MAC}

The 802.11 MAC employs a mandatory contention-based channel access scheme called Distributed Coordination Function (DCF), which is based on Carrier Sense Multiple Access with Collision Avoidance (CSMA/CA) and an optional centrally controlled channel access scheme called Point Coordination Function (PCF) [1]. With PCF, the time is divided into repeated periods, called SuperFrames (SFs), which consist of a Contention Period (CP) and a Contention Free Period (CFP). During the CP, the channel is accessed using the DCF whereas, during the CFP, is accessed using the PCF.

Recently, in order to support also delay-sensitive multimedia applications, such as real-time voice and video, the 802.11 e working group has enhanced the 802.11 MAC with improved functionalities. Four Access Categories (ACs), with different priorities, have been introduced. To satisfy the QoS requirements of each AC, the concept of TXOP (Transmission Opportunity) is introduced, which is defined as the time interval during which a station has the right to transmit and is characterized by a starting time and a maximum duration. The contiguous time during which TXOPs are granted to the same QSTA is called Service Period (SP).

Moreover, an enhanced access function, which is responsible for service differentiation among different ACs and is referred to as Hybrid Coordination Function (HCF), has been proposed [12]. The HCF is made of a contentionbased channel access, known as the Enhanced Distributed Coordination Access (EDCA), and of a HCF Controlled Channel Access (HCCA). The use of the HCF requires a centralized controller, which is called the Hybrid Coordinator (HC) and is generally located at the access point. Stations operating under 802.11e specifications are usually known as enhanced stations or QoS Stations (QSTAs).

The EDCA method operates as the basic DCF access method but using different contention parameters per access category. In this way, a service differentiation among ACs is statistically pursued. 
The HCCA method combines some of the EDCA characteristics with some of the PCF basic features (see Fig. 1). The time is partitioned into superframes; each of them starts with a beacon frame after which, for legacy purpose, there could be a contention free period for PCF access. The remaining part of the superframe forms the $\mathrm{CP}$, during which the QSTAs contend to access the radio channel using the EDCA mechanism.

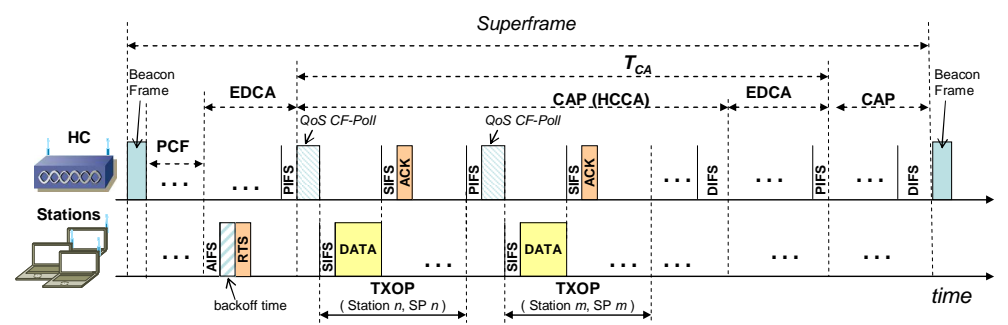

Fig. 1. Scheme of a superframe using the HCF controlled access method.

During the $\mathrm{CP}$, the $\mathrm{HC}$ can start a Contention Access Phase (CAP), in which only QSTAs, polled and granted with the QoS CF-Poll frame, are allowed to transmit during their TXOPs. Thus, the HC implements a prioritized medium access control. CAP length cannot exceed the value of the system variable dot11CAPLimit, which is advertised by the $\mathrm{HC}$ in the Beacon frame when each superframe starts [12].

According to IEEE 802.11e specifications, each QSTA can feed back queue length of each $\mathrm{AC}$ to the $\mathrm{HC}$ in each frame header. As shown in this paper, this information can be fruitfully exploited to design novel HCCA-based dynamic bandwidth allocation algorithms using feedback control theory. In fact, the 802.11e draft does not specify how to schedule TXOPs in order to provide the required QoS; it only suggests a simple scheduler which assigns fixed TXOPS using the static values declared in the Traffic Specifications (TSPECs) during the admission phase.

\subsection{Overview of the Power Saving in 802.11 infrastructure WLANs}

The power saving issue has been addressed in the 802.11 standard [1], by defining two different power states for a station: the Awake State in which the station is fully powered (i.e., the WNIC is on and consumes the power needed to transmit/receive frames and to sense the channel); the Doze State in which the station is not able to transmit or receive (i.e, the WNIC consumes very low power). Moreover, two power management modes have been introduced: the $A c$ tive Mode in which a station may receive frames at any time, i.e., it is always in awake state; the Power Save (PS) Mode in which a station is normally in the Doze State and enters in the Awake State to transmit frames and to receive beacon frames, broadcast, and unicast transmissions. 
The AP cannot transmit data to stations operating in PS mode, but it has to buffer frames and to transmit them only when such stations are in awake state.

In all beacon frames, the traffic indication message (TIM) is sent to indicate stations in PS mode (i.e., PS stations) which have buffered data in the AP. The buffered broadcast and multicast frames are signaled through the delivery TIM (DTIM) element.

A PS Station shall wake up in order to receive the Beacon frame and to detect if the AP has buffered frames for it.

If the PS station accesses the channel with the DCF method and there are pending data in the AP, during the Contention Period (CP) it sends to the AP a PS-Poll frame in order to receive buffered data, then can transit in doze state. Broadcast frames are sent immediately after the beacon frame that includes DTIM.

\subsection{IEEE 802.11e power saving enhancements}

The IEEE 802.11e Working Group have introduced a new power saving mechanism, known as Automatic Power Save Delivery (APSD) [12], which allows the delivery of downlink frames to a station according to a defined "schedule", i.e., the downlink frames are transmitted by the $\mathrm{HC}$ only in given service periods. In particular, when APSD is active, the HC buffers the data frames addressed to APSD stations (i.e., stations which use the APSD mode) in doze state and it transmits them according to two different type of service periods: Scheduled and Unscheduled. Scheduled service periods occur always at the same time instants in the superframe and they are assigned by the $\mathrm{HC}$ to a station when a new traffic stream starts. During its scheduled service period, a station is awake to receive buffered downlink frames and/or polls the HC. Unscheduled service periods are asynchronous in the superframe and they occur as soon as the HC knows that the APSD station wakes up by receiving any frame from the station.

\section{The PS FBDS Bandwidth Allocation Algorithm}

This section summarizes the FBDS bandwidth allocation algorithm proposed in [13] and introduces its power saving extension PS FBDS. The algorithm, which has been designed using classical feedback control theory, distributes the WLAN bandwidth among all the multimedia flows by taking into account the queue levels fed back by the QSTAs.

We will refer to a WLAN system made of an Access Point (AP) and a set of quality of service enabled mobile stations (QSTAs). Each QSTA has $N$ queues, with $N \leq 4$, one for any AC in the 802.11e proposal. Let $T_{C A}$ be the time interval between two successive CAPs. Every time interval $T_{C A}$, assumed constant, the AP must allocate the bandwidth that will drain each queue during the next CAP. We assume that at the beginning of each CAP, the AP is aware of all the queue levels $q_{i}, i=1, \ldots, M$ at the beginning of the previous CAP, where $M$ is the total number of traffic queues in the WLAN system. 
The dynamics of the $i^{\text {th }}$ queue can be described by the following discrete time linear model:

$$
q_{i}(k+1)=q_{i}(k)+d_{i}(k) \cdot T_{C A}+u_{i}(k) \cdot T_{C A}, \quad i=1, \ldots, M,
$$

where $q_{i}(k) \geq 0$ is the $i^{t h}$ queue level at the beginning of the $k^{t h} \mathrm{CAP} ; u_{i}(k) \leq 0$ is the average depletion rate of the $i^{\text {th }}$ queue (i.e., the bandwidth assigned to drain the $i^{t h}$ queue); $d_{i}(k)=d_{i}^{s}(k)-d_{i}^{C P}(k)$ is the difference between $d_{i}^{s}(k) \geq 0$, which is the average input rate at the $i^{\text {th }}$ queue during the $k^{t h} T_{C A}$ interval, and $d_{i}^{C P}(k) \geq 0$, which is the amount of data transmitted by the $i^{\text {th }}$ queue during the $k^{t h} T_{C A}$ interval using EDCA divided by $T_{C A}$.

The signal $d_{i}(k)$ is unpredictable since it depends on the behavior of the source that feeds the $i^{\text {th }}$ queue and on the number of packets transmitted during the contention periods. Thus, from a control theoretic perspective, $d_{i}(k)$ can be modelled as a disturbance. Without loss of generality, a piece-wise constant model for the disturbance $d_{i}(k)$ can be assumed: $d_{i}(k)=\sum_{j=0}^{+\infty} d_{0 j} \cdot 1\left(k-t_{j}\right)$, where $1(k)$ is the unitary step function, $d_{0 j} \in \mathbb{R}$, and $t_{j}$ is a time lag.

Due to this assumption, the linearity of the system (1), and the superposition principle that holds for linear systems, we will design the feedback control law by considering only a step disturbance: $d_{i}(k)=d_{0} \cdot 1(k)$.

\subsection{The control law}

We design a control law to drive the queuing delay $\tau_{i}$ experienced by each frame of the $i^{\text {th }}$ queue to a desired target value $\tau_{i}^{T}$, representing the QoS requirement of the $\mathrm{AC}$ associated to the queue. In particular, we consider the control law:

$$
u_{i}(k+1)=-k_{i} \cdot q_{i}(k)
$$

which gives the way to compute the $\mathcal{Z}$-transform of $q_{i}(k)$ and $u_{i}(k)$ as follows:

$$
Q_{i}(z)=\frac{z \cdot T_{C A}}{z^{2}-z+k_{i} \cdot T_{C A}} D_{i}(z) ; \quad U_{i}(z)=\frac{-k_{i} \cdot T_{C A}}{z^{2}-z+k_{i} \cdot T_{C A}} D_{i}(z)
$$

whit $D_{i}(z)=\mathcal{Z}\left[d_{i}(k)\right]$. From eq. (3)the system poles are $z_{p}=\frac{1 \pm \sqrt{1-4 k_{i} \cdot T_{C A}}}{2}$, which give an asymptotically stable system if and only if $\left|z_{p}\right|<1$, that is:

$$
0<k_{i}<1 / T_{C A}
$$

In the sequel, we will always assume that $k_{i}$ satisfies this asymptotic stability condition stated by (4).

To investigate the ability of the control system to provide a queuing delays approaching the target value $\tau_{i}^{T}$, we apply the final value theorem to Eq. (3). By considering that the $\mathcal{Z}$-transforms of the step function $d_{i}(k)=d_{0} \cdot 1(k)$ is $D_{i}(z)=d_{0} \cdot \frac{z}{z-1}$ the following results turn out:

$$
u_{i}(+\infty)=\lim _{k \rightarrow+\infty} u_{i}(k)=\lim _{z \rightarrow 1}(z-1) U_{i}(z)=-d_{0} ; \quad q_{i}(+\infty)=d_{0} / k_{i},
$$


which implies that the the steady state queueing delay is:

$$
\tau_{i}(+\infty)=\left|q_{i}(+\infty) / u_{i}(+\infty)\right|=1 / k_{i} .
$$

Thus, the following inequality has to be satisfied in order to achieve a steadystate delay smaller than $\tau_{i}^{T}$ :

$$
k_{i} \geq 1 / \tau_{i}^{T} .
$$

By considering inequalities (4) and (6) we obtain that the $T_{C A}$ parameter has to fulfill the following constraint:

$$
T_{C A}<\min _{i=1 . . M} \tau_{i}^{T} .
$$

\subsection{Implementation issues}

Starting from the allocated bandwidth $u_{i}$, if the $i^{\text {th }}$ queue is drained at data rate $C_{i}$, the assigned $T X O P_{i}$ is obtained by the following relation [13]:

$$
\operatorname{TXOP}_{i}(k)=\left|u_{i}(k) \cdot T_{C A}\right| / C_{i}+O
$$

where $\operatorname{TXOP}_{i}(k)$ is the TXOP assigned to the $i^{\text {th }}$ queue during the $k^{\text {th }}$ service interval and $O$ is the time overhead due to ACK packets, SIFS and PIFS time intervals (see Fig. 1). The extra quota of TXOP due to the overhead $O$ depends on the number of frames corresponding to the amount of data $\left|u_{i}(k) \cdot T_{C A}\right|$ to be transmitted. $O$ could be estimated by assuming that all frames have the same nominal size specified into the TSPEC.

The above bandwidth allocation is based on the implicit assumption that the sum of the TXOPs assigned to each queue is smaller than the maximum CAP duration, which is the dot11CAPLimit; this value can be violated when the network is saturated.

In this case, it is necessary to reallocate the TXOPs to avoid exceeding the CAP limit. Each computed $T X O P_{i}(k)$ is decreased by an amount $\triangle T X O P_{i}(k)$ proportional to $C_{i}$ and $T X O P_{i}(k)$ [13], in order to obtain a fair bandwidth assignment.

\subsection{Power Save FBDS}

To manage the power saving, at the beginning of each superframe, a station using PS FBDS wakes up to receive beacon frames. Then, if the HCCA method is used, it does not pass in doze state until it has received the QoS-Poll frame and the TXOP assignment from the HC. After the station has drained its queue according to the assigned TXOP, it will transit in doze state only if there are not new poll or data frames from the HC.

When the EDCA is used, the station wakes up as soon as any of its queues becomes not empty. In this case, the backoff timer is set to zero; thus, a wireless station will gain the access to the channel with a higher probability than stations using the classical EDCA. In the sequel, we will refer to this slightly modified version of the EDCA as Power Save EDCA (PS EDCA). 


\section{Performance Evaluation}

To test the effectiveness of PS FBDS, we have implemented the algorithm using the $n s-2$ simulator [16] and we have run computer simulations involving audio, video and FTP data transfers. We have considered a WLAN network shared by a mix of $3 \alpha$ audio flows encoded with the G.729 standard, $\alpha$ video flows encoded with the MPEG-4 standard, $\alpha$ encoded with the H.263 standard, and $\alpha$ FTP best effort flows. From each wireless node, a single data flow is generated. Main characteristics of the considered multimedia flows are summarized in Table 1.

In the $n s-2$ implementation the $T_{C A}$ is expressed in Time Units (TU), which in the 802.11 standard [1] are equal to $1024 \mu \mathrm{s}$. We assume a $T_{C A}$ of $29 \mathrm{TU}$. The proportional gain $k_{i}$ is set equal to $1 / \tau_{i}^{T}$. We have compared FBDS, PS FBDS, EDCA, and PS EDCA algorithms for different network loads, by varying the load parameter $\alpha$.

For what concern the power consumption parameters, we consider a RF Transceiver which has: Tx power of $393 \mathrm{~mW}, \mathrm{Rx}$ power of $357 \mathrm{~mW}$, stand-by power of $125 \mathrm{~mW}$, doze power of $33 \mu \mathrm{W}$. In the simulation each station has an initial energy equal to $10 \mathrm{~J}$. Stations hosting FTP flows do not use any power saving extensions. FTP flows are used to fill in the bandwidth left unused by flows with higher priority. Power saving mechanism are enabled after the first $15 \mathrm{~s}$ of warm-up period; thus, delays are evaluated without considering this warm-up time interval.

Fig. 2.a show the average value of the one-way packet delay experienced by the MPEG flows for various values of the load factor $\alpha$. It shows that both FBDS and PS-FBDS provide the smallest delays at high network loads (i.e., when $\alpha \geq 6$ ). The reason is that FBDS allocates the right amount of bandwidth to each flow by taking into account the transmitting queue levels of the wireless nodes, this allows a cautious usage of the WLAN channel bandwidth, so that QoS constraints are met also in the presence of a high number of competing flows.

Regarding the power saving issue, Figs. 2.b-2.d report the residual energy of a node hosting a MPEG traffic source for $\alpha=3,6$, and 9. When $\alpha=9$, i.e., at high network load, it is straightforward to note that a great energy saving can be achieved using PS FBDS. In fact, after $50 \mathrm{~s}$ of activity, PS FBDS leads to a total energy consumption less than $5 \mathrm{~J}$, whereas, with the other schemes, the energy consumption is larger than $8 \mathrm{~J}$. When $\alpha=3$, i.e., at low network load, PS HCCA and PS EDCA provide the same energy saving because, at low

Table 1. Main features of the considered multimedia flows.

\begin{tabular}{l|l|l|l|l} 
Type of flow & $\begin{array}{l}\text { Nominal (Maximum) } \\
\text { MSDU Size }\end{array}$ & $\begin{array}{l}\text { Mean (Maximum) } \\
\text { Rate }\end{array}$ & $\begin{array}{l}\text { Target } \\
\text { Delay }\end{array}$ \\
\hline \hline MPEG-4 HQ & $1536(2304)$ byte & $770(3300) \mathrm{kbps}$ & $40 \mathrm{~ms}$ \\
\hline H.263 VBR & $1536(2304)$ byte & $450(3400) \mathrm{kbps}$ & $40 \mathrm{~ms}$ \\
\hline G.729 VAD & $60(60)$ byte & $8.4(24) \mathrm{kbps}$ & $30 \mathrm{~ms}$
\end{tabular}


network load, almost all traffic is served during the CP using the EDCA. For $\alpha=6$ intermediate results are obtained. Thus PS FBDS allows energy saving while providing the same delay bounds of the original FBDS algorithm.

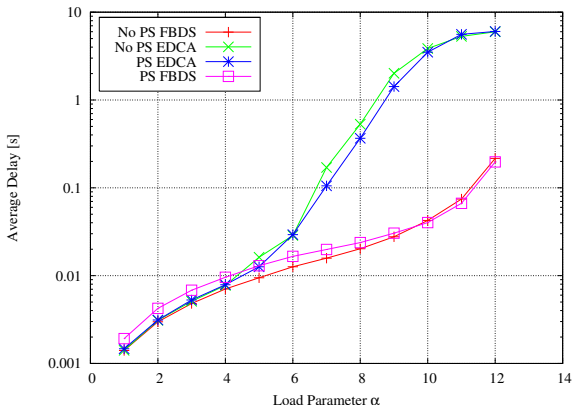

(a)

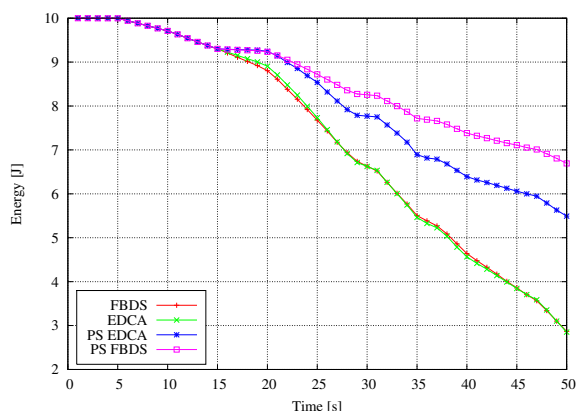

(c)

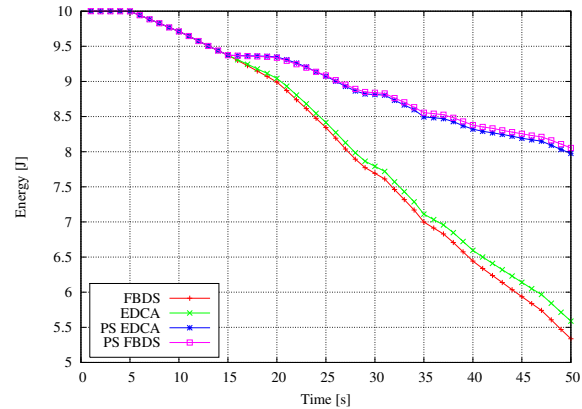

(b)

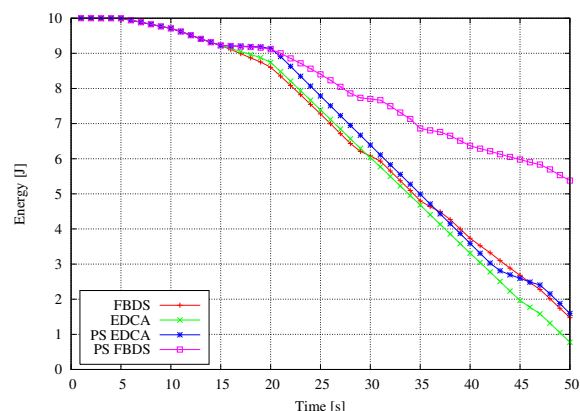

(d)

Fig. 2. Results for MPEG4 flows. (a) Average one-way delays vs. load parameter $\alpha$. Average Residual Energy using (b) $\alpha=3$; (c) $\alpha=6$; (d) $\alpha=9$.

Similar conclusions have been obtained for the H.263 traffic streams; results are not reported due to lack of space.

Results are very different when we consider G.729 flows (see Fig. 3). In fact, we have to consider that these flows are served with the maximum priority by the EDCA, and that the PS EDCA is more aggressive than standard EDCA.

Fig. 3.a shows that, when PS EDCA is used, the smallest delays are obtained. However, from these figures it can be noticed that delays provided by the other considered schemes are smaller than $100 \mathrm{~ms}$ also at high network loads, i.e., PS FBDS, FBDS, and standard EDCA provide acceptable performance.

Regarding energy consumption, Fig. 3.d reports the residual energy of a node hosting a G.729 traffic source when $\alpha=9$. While PS FBDS, FBDS and EDCA provide almost the same energy consumption as in the previous simulations, PS-EDCA enables the best energy saving. The reason is that the aggressiveness 
of PS EDCA allows stations hosting G.729 flows to listen the channel for very short time intervals before transmitting with an immediate impact on energy consumption. The gap between PS FBDS and PS EDCA diminishes for smaller values of $\alpha$ for the same reasons discussed above (see Figs. 3.b and 3.c).

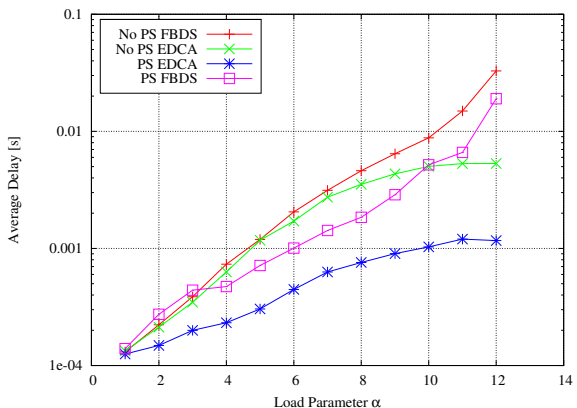

(a)

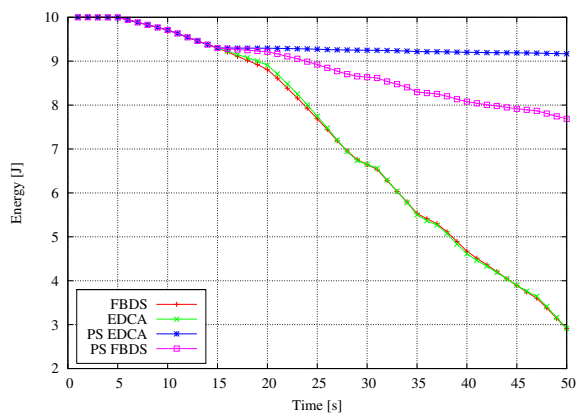

(c)

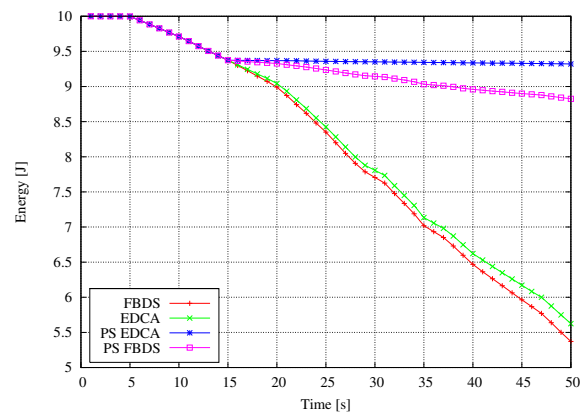

(b)

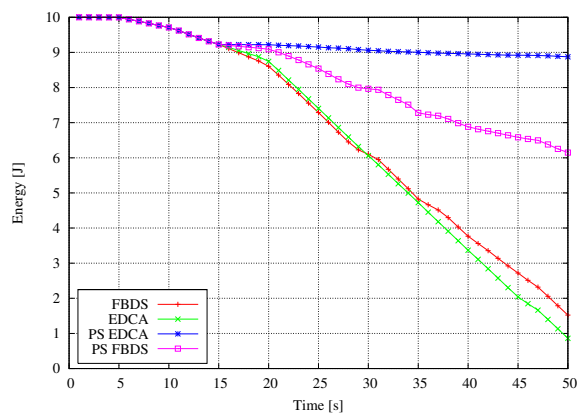

(d)

Fig. 3. Results for G.729 flows. (a) Average one-way delays vs. load parameter $\alpha$. Average Residual Energy using (b) $\alpha=3$; (c) $\alpha=6$; (d) $\alpha=9$.

\section{Conclusion}

In this paper, th PS FBDS scheduling algorithm has been proposed to achieve a good trade-off between power saving and QoS using 802.11e MAC. It has been designed using classical feedback control theory. Its performance has been investigated using $n s-2$ simulations in realistic scenarios where the wireless channel is shared by heterogeneous traffic flows. Simulation results show that PS FBDS is able to provide a bounded delay service to real-time flows and at the same time to significantly reduce energy consumption at both high and low network loads. 


\section{References}

1. IEEE 802.11: Information Technology - Telecommunications and Information Exchange between Systems Local and Metropolitan Area Networks Specific Requirements Part 11: Wireless LAN Medium Access Control (MAC) and Physical Layer (PHY) Specifications. ANSI/IEEE Std. 802.11, ISO/IEC 8802-11. First edn. (1999)

2. Baiamonte, V., Chiasserini, C.F.: An energy-efficient mac layer scheme for 802.11based wlans. In: Proceedings of the 2004 IEEE International Conference on Performance, Computing, and Communications. (2004) $689-694$

3. Woesner, H., Ebert, J.P., Schlager, M., Wolisz, A.: Power-saving mechanisms in emerging standards for Wireless LANs: the MAC level perspective. IEEE Personal Communications (1998) 40-48

4. Anastasi, G., Conti, M., Gregori, E., Passarella, A.: A performance study of powersaving polices for Wi-Fi hotspots. Computer Networks 45 (2004) 295-318

5. Choi, J.M., Ko, Y.B., Kim, J.H.: Enhanced Power Saving Scheme for IEEE 802.11 DCF Based Wireless Networks. In: Proceedings of the International Conference on Personal Wireless Communication (PWC'03). (2003) $835-840$

6. Anand, M., Nightingale, E.B., Finn, J.: Self-tuning Wireless Network Power Management. In: Proceedings of the ACM Mobicom 2003. (2003)

7. Jung, E., Vaidya, N.H.: An energy efficient MAC protocol for wireless LANs. In: Twenty-First Annual Joint Conference of the IEEE Computer and Communications Societies, ; INFOCOM 2002. Volume 3. (2002) 1756-1764

8. Bononi, L., Conti, M., Donatiello, L.: A Distributed Mechanism for Power Saving in IEEE 802.11 Wireless LANs. ACM/Baltzer Mobile Networks and Applications (MONET) 6 (2001)

9. Bruno, R., Conti, M., Gregori, E.: Optimization of Efficiency and Energy Consumption in p-Persistent CSMA-Based Wireless LANs. IEEE Transactions on Mobile Computing 1 (2002)

10. Yan, S., Zhuo, Y., Wu, S.: An Adaptive RTS Threshold Adjust Algorithm based on Minimum Energy Consumption in IEEE 802.11 DCF. In: Proceedings of the International Conference on Communication Technology (ICCT'2003). (2003) 12101214

11. Hsu, C.S., Sheu, J.P., Y.-C.Tseng: Minimize Waiting Time and Conserve Energy by Scheduling Transmissions in IEEE 802.11-based Ad Hoc Networks. In: Proceedings of the International Conference on Telecommunications (ICT 2003). (2003) 393399

12. IEEE 802.11 WG: Draft Amendment to Standard for Information Technology - Telecommunications and Information Exchange between Systems - LAN/MAN Specific Requirements - Part 11: Wireless Medium Access Control (MAC) and Physical Layer (PHY) Specifications: Medium Access Control (MAC) Quality of Service (QoS) Enhancements. IEEE 802.11e/D10.0. (2004)

13. Boggia, G., Camarda, P., Grieco, L.A., Mascolo, S.: Dynamic bandwidth allocation with call admission control for providing delay guarantees in IEEE 802.11e networks. Computer Communications, special issue 28 (2005) 325-337

14. Grilo, A., Macedo, M., Nunes, M.: A scheduling algorithm for QoS support in IEEE 802.11e networks. IEEE Wireless Communications (2003) 36-43

15. Ansel, P., Ni, Q., Turletti, T.: FHCF: A Fair Scheduling Scheme for 802.11e WLAN. Institut National de Recherche en Informatique et en Automatique (INRIA). (2003)

16. Ns-2: Network simulator. available at http://www.isi.edu/nsnam/ns (2004) 